\title{
Combination of selected mapping and statistical redistribution method for reducing peak to average power ratio in OFDM systems
}

\author{
A. A. Abdul Wahab and M. F. Ain \\ School of Electrical and Electronic Engineering, Universiti Sains Malaysia 14300 Nibong \\ Tebal, Pulau Pinang, Malaysia \\ Email: aeizaal@eng.usm.my
}

\begin{abstract}
Orthogonal frequency-division multiplexing (OFDM) is an attractive transmission technique for high-bit-rate communication systems. One major drawback of OFDM is the high peak-to-average power ratio (PAPR) of the transmitter's output signal. The selected mapping (SLM) approach provides good performance for PAPR reduction, but it requires a bank of inverse fast Fourier transforms (IFFTs) to generate a set of candidate transmission signals, and this requirement usually results in high computational complexity. In selected mapping (SLM) method a whole set of candidate signals is generated representing the same information, and then the most favourable signal as regards to PAPR is chosen and transmitted. SLM scheme is one of the initial probabilistic approaches for reducing the PAPR problem, with a goal of making occurrence of the peaks less frequent, not to eliminate the peaks. The scheme can handle any number of subcarriers and drawback associated with the scheme is the overhead of side information that needs to be transmitted to the receiver. This paper studies the problem of peak-to-average power ratio (PAPR) in orthogonal frequency division multiplexing (OFDM) system. It introduces a new PAPR reduction method that performs signal processing in the OFDM time domain together with the SLM method. The simple non-linear amplitude pre-distortion process of the proposed method changes the statistical characteristics of the OFDM symbol from Rayleigh distribution into Gaussian like distribution. This change in statistical distribution is accompanied by changes of the peak and average power values and consequently reduces in the PAPR value. For the QPSK OFDM system with 128 sub-carriers, $3 \mathrm{~dB}$ reduction in PAPR value is achieved. The proposed method shows that SLM with square rooting have higher PAPR reduction compare to the original SLM method. The approach is very flexible and can work with arbitrary number of sub-carries without restriction on the type of modulation applied on them.
\end{abstract}

Keywords: orthogonal frequency division multiplexing (OFDM), peak -to-average power ratio (PAPR), slm, statistical distribution.

\section{INTRODUCTION}

In the recent years a lot of advancements have been done in the multimedia technology and services. To enable these services with mobile communication high data rates and efficient usage of the available spectrum is required. Much research is on going to investigate transmission methods that can provide high data rates, can cope with multipath propagation, provide robustness against frequency selective fading or narrowband interference, and require less power and cost. Multicarrier modulation is one technique that provides us the desired demands of high data rates.

Orthogonal Frequency Division Multiplexing (OFDM) is a form of multicarrier modulation that can be seen either as modulation technique or a multiplexing scheme. OFDM is considered as a very promising candidate for future mobile communication systems. OFDM uses the Inverse Fast Fourier Transform (IFFT) operation to generate a large number of subchannels that are orthogonal. A cyclic prefix is added in the time domain that simplifies equalization and also eliminates interblock interference (IBI). OFDM is a widely used communication technique in broadband access applications requiring high data rates. It is already used in different WLAN standards (HIPERLAN 2, IEEE 802.11a), ADSL and digital video broadcasting (DVB). Even though OFDM has a number of advantages it has a potential drawback of high Peak to Average Power Ratio (PAPR). This high peak to average ratio causes nonlinearities in the transmitted signal and also degrades the power 
Am. J. Sci. Ind. Res., 2010, 1(2): 314-319

efficiency of the system. In order to reduce the PAR problem many researcher have made efforts and a large variety of different PAR reduction approaches are proposed (R.V. Nee and R. Prasad, 2000).

An OFDM signal is basically a bundle of narrowband carriers transmitted in parallel at different frequencies from the same source. In fact, this modulation scheme is often termed "multicarrier" as opposed to conventional "single carrier" schemes. Each individual carrier, commonly called a subcarrier, transmits information by modulating the phase and possible the amplitude of the subcarrier over the symbol duration. That is, each subcarrier uses either phase-shiftkeying (PSK) or quadrature-amplitudemodulation (QAM) to convey information just as conventional single carrier systems. However, OFDM or multi-carrier systems use a large number of low symbol rate subcarriers. The spacing between these subcarriers is selected to be the inverse of the symbol duration so that each subcarrier is orthogonal or non-interfering. This is the smallest frequency spacing that can be used without creating interference. At first glance it might appear that OFDM systems must modulate and demodulate each subcarrier individually. Fortunately, the well-known Fast Fourier transform (FFT) provides designers with a highly efficient method for modulating and demodulating these parallel subcarriers as a group rather than individually.

\section{MATERIALS AND METHODS}

\section{Orthogonal Frequency Division Multiplexing} (OFDM): The OFDM concept is based on spreading the data to be transmitted over a large number of carriers, each being modulated at a low rate. The carriers are made orthogonal to each other by appropriately choosing the frequency spacing between them: In contrast to conventional Frequency Division Multiplexing, the spectral overlapping among sub-carriers are allowed in OFDM since orthogonality will ensure the subcarrier separation at the receiver, providing better spectral efficiency and the use of steep bandpass filter was eliminated. OFDM transmission system offers possibilities for alleviating many of the problems encountered with single carrier systems. It has the advantage of spreading out a frequency selective fade over many symbols. This effectively randomizes burst errors caused by fading or impulse interference so that instead of several adjacent symbols being completely destroyed, many symbols are only slightly distorted (Bran, 1999): This allows successful reconstruction of majority of them even without forward error correction. Because of dividing an entire signal bandwidth into many narrow subbands, the frequency response over individual subbands is relatively flat due to subbands are smaller than coherence bandwidth of the channel. Thus, equalization is potentially simpler than in a single carrier system and even equalization may be avoided altogether if differential encoding is implemented. The orthogonality of subchannels in OFDM can be maintained and individual subchannels can be completely separated by the FFT at the receiver when there are no intersymbol interference (ISI) and intercarrier interference (ICI) introduced by the transmission channel distortion. Since the spectra of an OFDM signal is not strictly band limited, linear distortions such as multipath propagation causes each subchannel to spread energy into the adjacent channels and consequently cause ISI. One way to prevent ISI is to create a cyclically extended guard interval, where each OFDM symbol is preceded by a periodic extension of the signal itself. When the guard interval is longer than the channel impulse response or multipath delay, the ISI can be eliminated.

By using time and frequency diversity, OFDM provides a means to transmit data in a frequency selective channel. However, it does not suppress fading itself. Depending on their position in the frequency domain, individual subchannels could be affected by fading. This requires the use of channel coding to further protect transmitted data. Coded OFDM combined with frequency and time interleaving is considered the most effective means for a frequency selective fading channel (Shelswell, 1995). OFDM is a promising candidate for achieving high data rate transmission in mobile environment. It was shown to provide large improvements in BER performance in a Rayleigh Fading Environment. OFDM benefited from considerable research interest from the military applications and it had been used in several high frequency military systems such as KINEPLEX, ANDEFT and KATHRYN (Zou and Wu, 1995).Also the introduction of Digital Audio Broadcasting (DAB) based on OFDM, successfultest on OFDM for Digital Television Terrestrial Broadcasting (dTTb) and research on OFDM for HIPERLAN type II and Wireless ATM projects have increased the interested towards OFDM. For IMT2000/UMTS, two OFDM air interface concepts, Band Division Multiple Access (BDMA) and OFDM by Telia, were presented to Study Committee (Ojanpera, 1998). Digital Satellite services is becoming popular and application of OFDM to the Satellite Mobile Channel was proposed by (Weinstein and Ebert, 1996). OFDM used in DAB standard for CD- quality 
digital audio broadcast. One benefit of OFDM in a wireless communications system is that the receiver does not need to constantly adapt an equalizer as a single carrier system would. OFDM system shows much favourable properties such as high spectral efficiency, robustness to channel fading, immunity to impulse interference, capability of handling very strong echoes (multipath fading).The Orthogonal Frequency Division Multiplexing (OFDM) transmission scheme is the optimum version of the multicarrier transmission scheme. In the past, as well as in the present, the OFDM is referred in the literature Multi-carrier, Multi-tone and Fourier Transform. The concept of using parallel data transmission and frequency multiplexing was published in the mid 1960s. After more than thirty years of research and development, OFDM has been widely implemented in high speed digital communications. Due to recent advances of digital signal Processing (DSP) and Very Large Scale Integrated circuit (VLSI) technologies, the initial obstacles of OFDM implementation such as massive complex computation, and high speed memory do not exist anymore. The use of Fast Fourier Transform (FFT) algorithms eliminates arrays of sinusoidal generators and coherent demodulation required in parallel data systems and makes the implementation of the technology cost effective (Weinstein and Ebert, 1996).

Benefits of OFDM:The main benefits of OFDM compared to other multi-carrier techniques are:-

\section{Spectral Efficiency}

2. Resiliency to RF Interference

3. Lower Multi-Path Distortion

4. High Bit Rate Wireless Communications

5. Reducing ISI Probability

Disadvantages of OFDM: OFDM signals have a high Peak to Average Power Ratio (PAPR) causing RF devices to operate at a lower efficiency to avoid working in their non-linear region. OFDM systems also require a guard interval that causes a loss in power and bandwidth efficiency. Besides that, OFDM is sensitive to frequency offsets and phase noise. Frequency offsets occur when the voltage-controlled oscillator (VCO) at the receiver is not oscillating at exactly the same carrier frequency as the VCO in the transmitter. For the receiver, this offset between the two VCOs is seen as frequency translation in the signal and can lead to an increase in the error rate. While this is generally true for all modulations, OFDM is particularly sensitive to frequency offsets. In addition to the constant frequency offset discussed above, the frequency generated by a practical VCO tends to jitter, or vary, over time. To the receiver, this frequency variation looks like noise in the phase of the received signal and as a result this impairment is referred to as phase noise (Rohling, 1999).

Distribution of PAPR: An OFDM symbol consists of $N$ sub-carriers by the frequency spacing of $\Delta f$. The total bandwith $B$ will be divided into $N$ equally spaced sub-carriers with all sub-carriers are orthogonal to each other within a time interval of length $T=\frac{1}{\Delta f}$. Each sub-carrier can be modulated independently with complex modulation symbol $X_{m, n}$, where $\mathrm{m}$ is a time index and $\mathrm{n}$ is a sub-carrier index.

The m-th OFDM block period can be described by equation (1) as :

$$
x_{m}(t)=\frac{1}{\sqrt{N}} \sum_{n=0}^{N-1} X_{m, n} g_{n}(t-m T)
$$

Where, $g_{n}(t)$ is defined through equation (2).

$$
g_{n}(t)=\left\{\begin{array}{l}
\exp (j 2 \pi n \Delta f t), 0 \leq t \leq T \\
0, \text { else }
\end{array}\right.
$$

Where $g_{n}(t)$ is a rectangular pulse applied to each subcarrier (Han and Lee, 2005). The total continuous time signal $x(t)$ consisting of all the OFDM block is given by equation (3).

$$
x(t)=\frac{1}{\sqrt{N}} \sum_{m=0}^{\infty} \sum_{n=0}^{N-1} X_{m, n} g_{n}(t-m T)
$$

Consider a single OFDM symbol $(m=0)$ without loss of generality. This can be shown because there is no overlap between different OFDM symbols.

Since $m=0, X_{m, n}$ can be replaced by $X_{n}$. Then, the OFDM signal can be described as follows,

$$
x(t)=\frac{1}{\sqrt{N}} \sum_{n=0}^{N-1} X_{n} e^{j 2 \pi m \Delta f t}
$$

If the bandwith of the OFDM signal is $B=N \times \Delta f$ and the signal $x(t)$ is sampled by the sampling time of 
$\Delta t=\frac{1}{B}=\frac{1}{N \Delta f}$, then the OFDM signal is in discrete time form and can be written as shown in equation (5).

$$
x_{k}=\frac{1}{\sqrt{N}} \sum_{n=0}^{N-1} X_{n} e^{\frac{j 2 \pi k n}{N}}, k=0,2, \ldots N-1
$$

For an OFDM signal with $N$ subcarriers, the PAPR can be defined as

$$
P A P R=\frac{\max |x(t)|^{2}}{E|x(t)|^{2}}
$$

In particular, a baseband OFDM signal with $N$ subchannels has

$$
P A P R_{\max }=10 \log _{10} N
$$

From the central limit theorem, it follows that for large values of $N(N>64)$, the real and imaginary values of $x(t)$ become Gaussian distributed. Therefore the amplitude of the OFDM signal has a Rayleigh distribution, with a cumulative distribution given by

$$
F(z)=1-e^{-z}
$$

The probability that the PAPR is below some threshold level can be written as

$$
P(P A P R \leq z)=\left(1-e^{-z}\right)^{N}
$$

In fact, the complementary cumulative distribution function (CCDF) of PAPR of an OFDM is usually used, and can be expressed as

$$
P(P A P R>z)=1-\left(1-e^{-z}\right)^{N}
$$

\section{Selected Mapping Technique (SLM)}

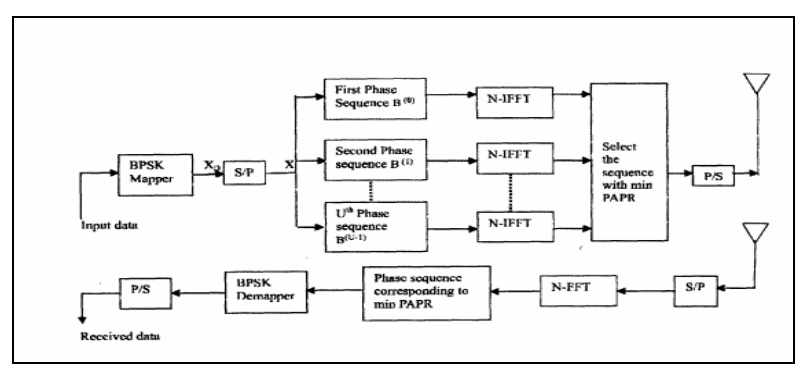

Fig 1: Block Diagram of SLM technique
The block diagram of the OFDM system with SLM technique is shown in Figure 1. At first, BPSK modulated input data is partitioned into a data block $X$ of length $N$. Then the data block is multiplied element by element with phase sequences $B^{(U)}=\left[b_{u, 0}, b_{u, 1}, \ldots b_{u, N-1}\right]^{T}, u=1,2, \ldots, U$ to make the $U$ phase rotated OFDM data blocks $X^{(U)}=\left[X_{u, 0}, X_{u, 1}, \ldots X_{u, N-1}\right]^{T}, u=1,2, \ldots, U \quad$ where $X_{u, m}=X_{m} b_{u, m}, m=0,1,2, \ldots, N-1$. All $U$ phase rotated OFDM data blocks represent the same information as the unmodified OFDM data block provided that the phase sequence is known. To include unmodified OFDM data block in the set of the phase rotated OFDM data blocks, the first phase sequence $B^{(0)}$ is set as all one vector of length $N$ (Han and Lee, 2005). After applying the SLM technique to $X,(4)$ becomes

$$
x^{(u)}(t)=\frac{1}{\sqrt{N}} \sum_{n=0}^{N-1} X_{n} b_{u, n} e^{j 2 \pi n \Delta f t}
$$

Subcarrier Statistical Redistribution: Some of the statistical properties of signals can be changed by applying special mathematical operations. As an example, the Rayleigh distribution of any signal will change into Gaussian distribution if the square root operation is applied to that signal. Also, the ChiSquare distribution can be transformed into Rayleigh distribution by applying the same process, square rooting (Wisam, Ali, Khatun and Jamalullail, 2007). Not only the statistical distribution changes by square rooting, but the mean and variance values of the signal are also varied. This process, square rooting, is exploited in this work to realize reduction of the PAPR value. From the central limit theorem and for large number of input samples, the imaginary and real parts of the IFFT outputs will follow Gaussian distributions. Hence, the amplitude (envelope) of the complex valued OFDM symbols will have Rayleigh distribution, and it is computed by ( $\mathrm{Wu}$ and Zou, 1995);

$$
|x(n)|=\sqrt{(\operatorname{Re}\{x[n]\})^{2}+(\operatorname{Im}\{x[n]\})^{2}}
$$

$|x(n)|:$ Amplitude values of OFDM symbols, random variable $(R V)$ of Rayleigh distribution.

$(\operatorname{Re}\{x[n]\})$ : Real part of OFDM symbols, RV of Gaussian distribution. 
$(\operatorname{Im}\{x[n]\})$ : Imaginary part of OFDM symbols, RV of Gaussian distribution.

At the same time, the power distribution becomes a central chi-square distribution with two degrees of freedom (Nee and Prasad, 2000).

$$
|x(n)|^{2}=(\operatorname{Re}\{x[n]\})^{2}+(\operatorname{Im}\{x[n]\})^{2}
$$

Where $|x(n)|^{2}$ denotes the power value of OFDM output symbol which is exponentially distributed with mean $2 \sigma^{2}$. The discrete form of the conventional OFDM symbol, as depicted in Figure 1, is processed in the proposed method by the following formula before transferring to the D/A:

$$
\tilde{x}_{k}=\sqrt{\left|x_{k}\right|} e^{j \phi_{k}}
$$

where $\phi_{k}$ is the phase of $x_{k}$.

By examining (12) and (13), we see that the relationship between the two random variables are reciprocal, therefore we could easily convert between the two by applying square operation on their samples, or square rooting for opposite conversion. This will in turn change both the statistical distribution and describing parameters of the processed signals. The changes inflicted on the mean and variance values of the processed signals are reflected directly on the peak and average power values. The impact of this operation on the average power value is higher than that on the peak power value, which always leads to reduction in the PAPR value (Tiku, 1982).

\section{RESULTS AND DISCUSSION}

In this work, computer simulation is implemented to evaluate the performance of the proposed method. In our simulation, we assume an OFDM system with $\mathrm{N}=128$ sub-carriers together with QPSK modulation scheme. The PAPR reduction efficiency of the proposed method is shown in Figure 2. The results are presented in terms of complementary cumulative distribution function (CCDF) for an OFDM output symbol blocks before and after processing. The difference in PAPR performance between the conventional OFDM system, and that obtained from the SLM and the new method is clearly observed. Figure 2 shows the CCDF of both conventional OFDM system and those after the addition of the proposed method. As could be seen, the performance of the system in terms of PAPR show that maximum reduction in PAPR value of slightly more than $1.8 \mathrm{~dB}$ was achieved by the SLM method for the OFDM system parameters of 128 samples and QPSK modulation scheme. The influence of square rooting method after the SLM process on the PAPR reduction of the OFDM system is also considered. From Figure 2 more than $5 \mathrm{~dB}$ reduction in PAPR value was achieved compare to $1.8 \mathrm{~dB}$ if only using the SLM method. The result proves that the square rooting method will reduce another $3 \mathrm{~dB}$ of PAPR value from the conventional OFDM system. The PAPR reduction efficiency will be tested using analysis of variance. The analysis of variance is used to determine whether a factor affects the response variable (Tiku, 1982). In this case, the factor is SLM method and time domain statistical method. If the factor is significant, the mean response differs for the various treatments. The box plot in Figure 3, indicate that the original OFDM has higher and more variable PAPR value than the other methods. The new method seems to have the lowest PAPR value than the other methods. To test whether the mean of the PAPR value differ, the null and alternative hypotheses are,

$$
\begin{aligned}
& H_{0}: \mu_{1}=\mu_{2}=\mu_{3} \\
& H_{1}: \mu_{1} \neq \mu_{2} \neq \mu_{3}
\end{aligned}
$$

Where $\mu_{1}$ is the mean of PAPR for original OFDM, $\mu_{2}$ is the mean value of PAPR for clipping method and $\mu_{3}$ for the new method.

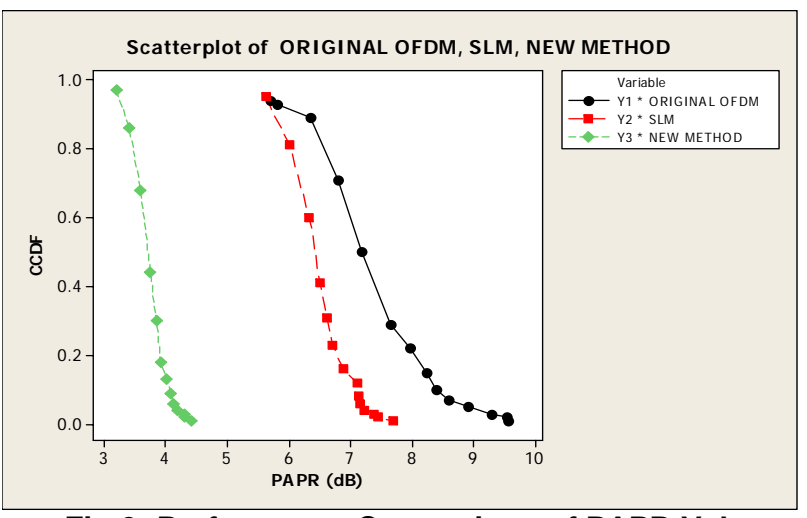

Fig 2: Performance Comparison of PAPR Value 


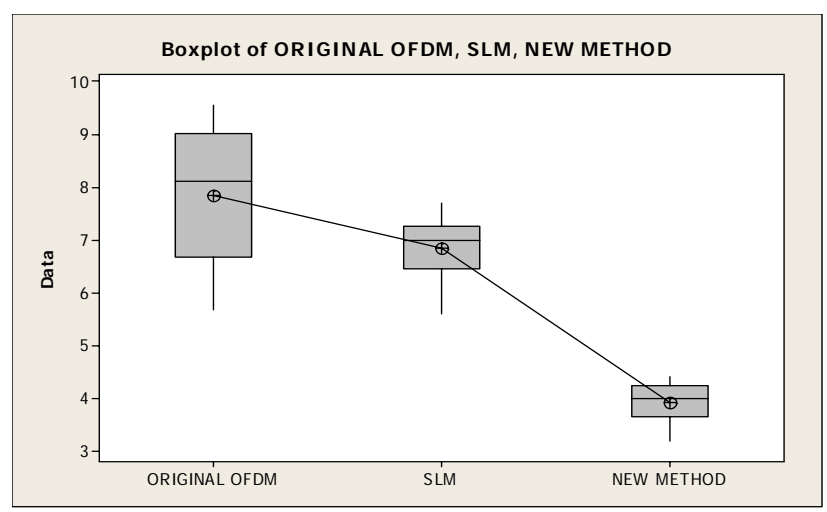

Fig 3: Box Plot Comparison of PAPR Value

From Table 1, the test statistic, $F=73.42$ has a $p$ value of 0.000 . The $p$-value is the probability of obtaining a test statistics as large as $F=73.42$, assuming $H_{0}$ is true. Since the p-value is less than confidence level $\alpha=0.05$, the null hypothesis is rejected. The confidence intervals from Table 2 indicate which mean may differ and from the result, the confidence interval for new method does not overlap with other confidence interval. It appears that the new method has the lowest mean of PAPR value.

Table 1: One-Way ANOVA

\begin{tabular}{|c|c|c|c|c|c|}
\hline Source & DF & SS & MS & F & P \\
\hline Factor & 2 & 110.945 & 55.473 & 73.42 & 0.000 \\
\hline Error & 38 & 28.711 & 0.756 & & \\
\hline Total & 40 & 139.656 & & & \\
\hline
\end{tabular}

Table2: Confidence Interval Comparison

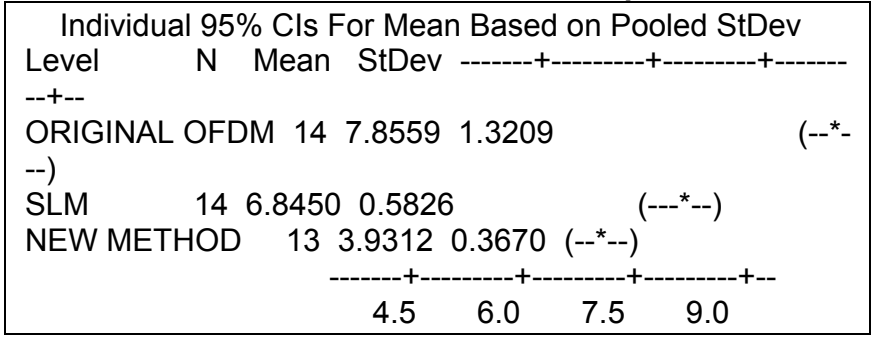

CONCLUSIONS: A new PAPR reduction method for the OFDM system by combining both SLM method and square rooting has been proposed and investigated. This was achieved by adding the time domain statistical method after the selected mapping process. This process involves the square rooting method that changes the effect on the mean and variance values of the processed signals. The proposed method is suitable for OFDM applications that are sensitive to spectral efficiency and noise, since it allows reduction in PAPR value with no out-of band radiation. For QPSK OFDM system with $\mathrm{N}=128$ data sub-carriers, more than $5 \mathrm{~dB}$ reduction in PAPR value was achieved compare with the conventional OFDM system.

\section{REFERENCES}

R.V. Nee and R. Prasad, "OFDM for Wireless Multimedia Communications, "Artech House, 2000.

Shelswell, P., "The COFDM Modulation System: The Heart of Digital Audio Broadcasting", Electronics \& Communication Engineering Journal, pp. 127-135, 1995.

"Broadband Radio Access Network (BRAN); High Performance Radio Local Area Network (HIPERLAN) Type 2; Requirements and Architecture for Wireless Broadband Access," TR101031, 1999.

S.H. Han and J.H. Lee, "An Overview of Peak to Average Power Ratio Reduction Techniques for Multicarrier Transmission," IEEE Wireless Comm., Vol. 12, No. 2, April 2005, pp. 56-65.

Rohling H., "Broadband OFDM Radio Transmission for Multimedia Applications," IEEE Proceedings, Vol. 87, pp. 1778-1788, 1999.

May, T. and Rohling, H., "Reducing the Peak to Average Power Ratio of OFDM and Radio Transmission System," IEEE Vehicular Technology Conference, Vol. 3, 1998.

Wu,Y. abd Zou, W. Y., "Orthogonal Frequency Division Multiplexing: A Multicarrier Modulation Scheme," IEEE Transactions on Consumer Electronics, Vol. 41, No. 3, pp. 392-399, 1995.

Tiku, M. L., "Robust Statistics for Testing Equality of Means and Variances," Communications in Statistics, Theory and Methods, pp. 2543-2558, 1982.

Wisam F. Azzo, M. Ali, S. Khatun and S. Jamalullail, "Time Domain Statistical Control for PAPR Reduction in OFDM System," Proceedings of Asia-Pacific Conference on Communications, 2007.

Zou, W.Y. and Wu, Y., "COFDM: An Overview," IEEE Transactions on Broadcasting, Vol. 41, pp. 1-8, 1995.

Ojanpera, T., "An Overview of Air Interface Multiple Access for IMT-2000/UMTS," IEEE Communications Magazine, pp. 82-95, 1998.

Weinstein, S.B. and Ebert, P.M., "Data Transmission by Frequency Division Multiplexing Using the Discrete Fourier Transform," IEEE Transactions on Communication Technology, Vol. 19, pp. 628-634, 1996. 